\title{
Comparison of FOLFOX and DOF regimens as first-line treatment in East Asian patients with advanced gastric cancer
}

This article was published in the following Dove Press journal: OncoTargets and Therapy

\section{Mengyao Liu',2 \\ Guofang $\mathrm{Hu}^{2}$ \\ Yuan Wang ${ }^{2}$ \\ Jun Guo ${ }^{2}$ \\ Liyan Liu² \\ Xiao $\mathrm{Han}^{2}$ \\ Zhehai Wang ${ }^{2}$}

'School of Medicine and Life Sciences, University of Jinan-Shandong Academy of Medical Sciences, Jinan, Shandong, ${ }^{2}$ Department of Oncology, Shandong Cancer Hospital, affiliated to Shandong University, Shandong Academy of Medical Sciences, Jinan, Shandong, People's Republic of China
Background: Our study retrospectively assesses the safety and efficacy of the FOLFOX (oxaliplatin, fluorouracil, and leucovorin) versus DOF (docetaxel, oxaliplatin, and fluorouracil) regimens in untreated locally advanced gastric cancer (AGC).

Patients and methods: A total of 108 patients underwent DOF $(\mathrm{N}=58)$ and FOLFOX $(\mathrm{N}=50)$ regimens. The end points were overall response rate (ORR), survival, and toxicity. Kaplan-Meier curve was used to estimate overall survival (OS) and progression-free survival (PFS) and Cox regression for multivariate analysis.

Results: The ORRs were 50\% for DOF and 30\% for FOLFOX groups $(P<0.05)$, and disease control rates were $91.4 \%$ and $72 \%$, respectively. The median PFS and OS in DOF group were significantly better than FOLFOX group ( 8.2 versus 6.4 months, $P<0.05 ; 16.3$ versus 11.2 months, $P<0.001)$. Both groups showed acceptable toxicity; all grades and grade 3-4 toxicity had no significant differences $(P=0.071 ; P=0.247)$. However, the incidence of grade $3-4$ peripheral neuropathy was significantly higher in DOF group $(10.3 \%$ versus $2 \%, P<0.05)$. In the subgroup analysis for elderly AGC patients ( $\geq 65$ years), administration of DOF also resulted in a superior PFS ( 8.5 versus 5.9 months; $P=0.038$ ) and OS ( 15.3 versus 9.8 months; $P=0.004$ ) compared with FOLFOX. However, DOF regimen was associated with more neutropenia $(67 \%$ versus $30 \% ; P<0.05$ ), thrombocytopenia ( $61 \%$ versus $52 \% ; P<0.05$ ), and peripheral neuropathy (49\% versus $22 \% ; P<0.05$ ).

Conclusion: DOF regimen was more effective than FOLFOX for AGC, both in younger and older patients. The adverse effects of the two regimens were manageable. The combination of docetaxel/oxaliplatin/fluorouracil was active and well tolerated in AGC patients and deserves further evaluation. However, for elderly patients with AGC, the DOF regimen was associated with worse toxicities; therefore, the FOLFOX regimen might be a more suitable option.

Keywords: East Asian AGC patients, chemotherapy, safety, efficacy

\section{Introduction}

Gastric cancer continues to be one of the most common causes of cancer-related mortality worldwide, ranking as the third and fifth causes of cancer death in men and women, respectively. The highest incidence rates of gastric cancer are in Eastern Asia, Eastern Europe, and South America. ${ }^{1,2}$ The overall survival (OS) of advanced gastric cancer (AGC) remains poor; the median OS was reported as $\sim 7.5-12$ months versus 3-5 months in patients receiving chemotherapy, compared with those accepting best supportive care alone. Although the overall treatment result was not optimistic, systemic chemotherapy has been associated with a remarkably superior survival and a better quality of life compared with supportive care..$^{3-6}$ In addition, research and meta-analysis
Correspondence: Zhehai Wang Department of Oncology, Shandong Cancer Hospital, Shandong University, 440 Jiyan Road, Jinan, Shandong 250I 17 , People's Republic of China Tel/fax +8653167626331 Email wangzhehai7788@sina.com
OncoTargets and Therapy 2018:1| 375-38|

375 
studies have shown that combination chemotherapy is quite effective, ${ }^{7-9}$ and a regimen of three drugs seems to be more advantageous than monotherapy or doublet therapy. ${ }^{10-11}$

The combination of docetaxel, cisplatin, and infused fluorouracil (5-FU; DCF) regimen was compared to the cisplatin and 5-FU (CF) regimen in the V325 trial. The median time to progression (TTP) (5.6 months versus 3.7 months; $P<0.001$ ) and OS (9.2 months versus 8.6 months; $P=0.02)$ and 1 -year survival (40\% versus 32\%; $P<0.05$ ) were significantly longer for the DCF than the CF regimen. ${ }^{11}$ Follow-up research reported improvements in both clinical benefit and quality of life. ${ }^{12,13}$ However, the DCF regimen was associated with significant hematological toxicity, and grade 3-4 treatmentemergent adverse events occurred in $69 \%$ of patients. ${ }^{11}$ Therefore, more effective chemotherapeutic regimens with reduced toxicity need to be found.

Oxaliplatin has been shown to be better tolerated than cisplatin, especially in older patients, ${ }^{14,15}$ and has proven efficacy in combination with 5-FU. ${ }^{16,17}$ Therefore, the combination chemotherapy of 5-FU with oxaliplatin has been studied in numerous Phase II studies, using different doses and schedules, and the outcomes were satisfactory. ${ }^{18,19}$

During the last few decades, the FOLFOX (oxaliplatin, fluorouracil, and leucovorin) regimen has been reported to have considerable antitumor activity and tolerable toxicity in patients with AGC in several Phase II clinical trials. ${ }^{20-23}$ Considering these findings, we substituted oxaliplatin for cisplatin in the DCF regimen and retrospectively compared the efficacy and toxicity of DOF versus FOLFOX regimens as the first-line treatment in patients with untreated locally AGC.

\section{Patients and methods}

A total of 108 patients with locally AGC with complete data in the Shandong Cancer Hospital from January 2013 to December 2014 were enrolled in this retrospective study, and 58 and 50 patients underwent the DOF or FOLFOX regimen as first-line chemotherapy, respectively. The medical data accessed were made anonymous. These patients were nonconsecutively allocated to one of two chemotherapy regimens by physician's preference. We compared the progression-free survival (PFS), OS, and various clinical variables, including prior treatment characteristics, major toxicities (mainly grade 3-4 hematological adverse effects), and response to chemotherapy. The end points were response rate, survival, and toxicity. The patients were required to satisfy the following criteria: 1) aged 18-75 years at diagnosis; 2) a histologically proven unresectable advanced or metastatic lesion; 3) an Eastern Cooperative Oncology Group
(ECOG) performance status $\leq 2$; 4) FOLFOX or DOF regimen as first-line chemotherapy for at least two cycles; 5) no active concomitant malignancy; 6) adequate organ function; and 7) complete and retrievable clinical medical records. The study was approved by the Medical Ethics Committee of the Shandong Cancer Hospital. As the study data were obtained by reviewing medical records, the Medical Ethics Committee of the Shandong Cancer Hospital did not require that participant consent be obtained, although the authors confirm that all patients had signed a written informed consent before treatment.

\section{Treatment}

A total of 108 patients with AGC were enrolled, and 58 patients received DOF regimen $\left(75 \mathrm{mg} / \mathrm{m}^{2}\right.$ docetaxel was administered by 1 -hour infusion and $85 \mathrm{mg} / \mathrm{m}^{2}$ oxaliplatin by 2 -hour intravenous infusion on day 1 , then $750 \mathrm{mg} / \mathrm{m}^{2}$ 5 -FU was infused on days 1-5) every 21 days. In contrast, 50 patients received the FOLFOX regimen (oxaliplatin $85 \mathrm{mg} / \mathrm{m}^{2}$ and leucovorin $400 \mathrm{mg} / \mathrm{m}^{2}$ were administered as an intravenous infusion, followed by a 5 -FU bolus of $400 \mathrm{mg} / \mathrm{m}^{2}$ and 5 -FU $2,400 \mathrm{mg} / \mathrm{m}^{2}$ as a 46 -hour continuous infusion) every 14 days. Complete blood counts were performed before and after every cycle of chemotherapy. In cases of severe hematological toxicity, dose adjustment was implemented in the next chemotherapy cycle. If the white blood cell count was under $3,000 / \mathrm{mm}^{3}$ after the chemotherapy, the patient was treated with recombinant human granulocyte colony-stimulating factor (G-CSF) injection 48-72 hours following completion of chemotherapy. If the white blood cell count decreased to $2,000-2,500 / \mathrm{mm}^{3}$, the dose of docetaxel, 5-FU, and oxaliplatin was temporarily decreased by $50 \%$ at the beginning of the next course of chemotherapy. In the event of grade 4 hematological or grade 3-4 gastrointestinal reaction, the doses were reduced by $25 \%$ in the subsequent course. Chemotherapy was discontinued until disease progression, unacceptable toxicity, patient refusal, or the physician's decision.

\section{Response evaluation}

Response evaluation was performed every 8-12 weeks, based on the Response Evaluation Criteria in Solid Tumors (RECIST) 1.1 guidelines. ${ }^{24}$ The status of response to treatments was classified into complete response (CR), partial response (PR), stable disease (SD), and progressive disease. In addition, overall response rate (ORR) was defined as CR plus PR, and disease control rate (DCR) was defined as CR plus PR and SD. Toxicities were evaluated according to the 
National Cancer Institute Common Terminology Criteria for Adverse Events, version 4.0. ${ }^{25}$ Peripheral neuropathy was graded according to the oxaliplatin-specific scale: grade 1, paresthesias or dysesthesias of short duration with complete recovery before the next cycle; grade 2, paresthesias persisting for two cycles without functional impairment; and grade 3, permanent paresthesias interfering with function. ${ }^{14}$

\section{Statistical analysis}

The baseline characteristics were compared by the chi-square test (for categorical variables) or the two-sample $t$-test (for continuous variables). The Kaplan-Meier method was used to analyze the median OS and PFS and draw survival curves, and the Cox-regression model was used for multivariate analysis of independent prognostic factors of AGC. A value of $P<0.05$ was considered to be statistically significant. All statistical analyses were performed using the IBM SPSS software (Chicago, IL, USA), version 17.0. Safety analysis was conducted using descriptive statistics.

\section{Results}

\section{Patient characteristics}

The baseline characteristics were well balanced between the two groups in this study except for sex $(P=0.045)$, and also the FOLFOX group had a higher ECOG performance status compared with the DOF group $(P=0.044)$ (Table 1). The median age of patients was 58 years (range 34-79 years), and among them 32 (29.6\%) were female. In the DOF group, 21 patients were older than 65 years $(36.2 \%)$ and in the FOLFOX group, 12 patients (24\%). Twenty-eight patients (25.9\%) had stage III disease and 80 (74.1\%) had stage IV disease. According to the ECOG performance status scale, $2(3.4 \%)$ of the patients in the DOF group and $8(16 \%)$ in the FOLFOX group had a PS of 2 . The usual treatment course was four to six cycles.

\section{Response rate}

The response rates in the two groups are shown in Table 2. The ORR was $50 \%$ for the DOF and $30 \%$ for the FOLFOX group $(P<0.05)$. The DCR was $91.4 \%$ for the DOF and $72 \%$ for the FOLFOX group $(P<0.05)$.

\section{Survival}

As shown in Figures 1 and 2, the median PFS was longer in the DOF compared with the FOLFOX group (8.2 versus 6.4 months, $P=0.011$ ), and the median OS also showed a similar trend (16.3 versus 11.2 months, $P<0.001)$. In the subgroup analysis, for AGC patients older than 65 years, the
Table I Patient characteristics

\begin{tabular}{|c|c|c|c|}
\hline \multirow[t]{2}{*}{ Characteristics } & \multicolumn{2}{|c|}{ Chemotherapeutic regimen } & \multirow[t]{2}{*}{$P$-value } \\
\hline & $\begin{array}{l}\text { DOF, N=58 } \\
\text { n (\%) }\end{array}$ & $\begin{array}{l}\text { FOLFOX, N=50 } \\
\text { n (\%) }\end{array}$ & \\
\hline Number of patients & $58(53.7)$ & $50(46.3)$ & \\
\hline Age (years) & & & 0.053 \\
\hline Median, mean \pm SD & $61 \pm 7.4$ & $56 \pm 8.5$ & \\
\hline$<65$ & $37(63.8)$ & $38(76)$ & \\
\hline$\geq 65$ & $21(36.2)$ & $12(24)$ & \\
\hline Sex & & & 0.045 \\
\hline Male & $42(72.4)$ & $44(88.0)$ & \\
\hline Female & $16(27.6)$ & $6(12.0)$ & \\
\hline ECOG & & & 0.044 \\
\hline 0 & $34(58.6)$ & $30(60)$ & \\
\hline I & $22(37.9)$ & $12(24)$ & \\
\hline 2 & $2(3.4)$ & $8(16)$ & \\
\hline Clinical stage & & & 0.370 \\
\hline III & $13(22.4)$ & $15(30)$ & \\
\hline IV & $45(77.6)$ & $35(70)$ & \\
\hline Tumor location & & & 0.129 \\
\hline Cardia & $18(3 \mid .0)$ & $23(46)$ & \\
\hline Gastric body & $14(24.1)$ & $9(18)$ & \\
\hline Fundus of stomach & $3(5.2)$ & $6(12)$ & \\
\hline Antrum & $20(34.5)$ & $12(24)$ & \\
\hline Pylorus & $3(5.2)$ & $0(0)$ & \\
\hline Metastasis & & & 0.06 \\
\hline None & $13(22.4)$ & $15(30)$ & \\
\hline Abdominal cavity & $20(34.5)$ & $12(24)$ & \\
\hline Lung & $2(3.4)$ & $9(18)$ & \\
\hline Liver & $18(3 \mid)$ & $9(18)$ & \\
\hline Bone & $5(8.6)$ & $5(10)$ & \\
\hline Ascites & $14(24.1)$ & $5(10)$ & 0.054 \\
\hline
\end{tabular}

Abbreviations: DOF, docetaxel, oxaliplatin, and fluorouracil; ECOG, Eastern Cooperative Oncology Group performance status; FOLFOX, oxaliplatin, fluorouracil, and leucovorin.

DOF regimen also resulted in a significantly superior PFS (8.5 versus 5.9 months; $P=0.038$ ) and an $\mathrm{OS}(15.3$ versus 9.8 months; $P=0.004$ ) compared with the FOLFOX regimen.

\section{Multivariate analyses}

The multivariate Cox proportional hazard regression analysis for OS showed that regimen was an independent prognostic factor $(95 \%$ CI 1.474-4.092, $P=0.001)$. We also analyzed all the aforementioned factors and found that response,

Table 2 Tumor response to treatment

\begin{tabular}{llll}
\hline Characteristics & \multicolumn{2}{l}{ Chemotherapeutic regimen } & P-value \\
\cline { 2 - 3 } & $\begin{array}{l}\text { DOF, } \mathbf{N}=\mathbf{5 8} \\
\mathbf{n}(\%)\end{array}$ & $\begin{array}{l}\text { FOLFOX N=50 } \\
\text { n (\%) }\end{array}$ & \\
\hline CR & $3(5.2)$ & I (2) & 0.036 \\
PR & $26(44.8)$ & I4 (28) & \\
SD & $24(4 I .4)$ & $2 I(42)$ & \\
PD & $5(8.6)$ & I4 (28) & \\
\hline
\end{tabular}

Abbreviations: $C R$, complete response; DOF, docetaxel, oxaliplatin, and fluorouracil; FOLFOX, oxaliplatin, fluorouracil, and leucovorin; PD, progressive disease; $\mathrm{PR}$, partial response; SD, stable disease. 


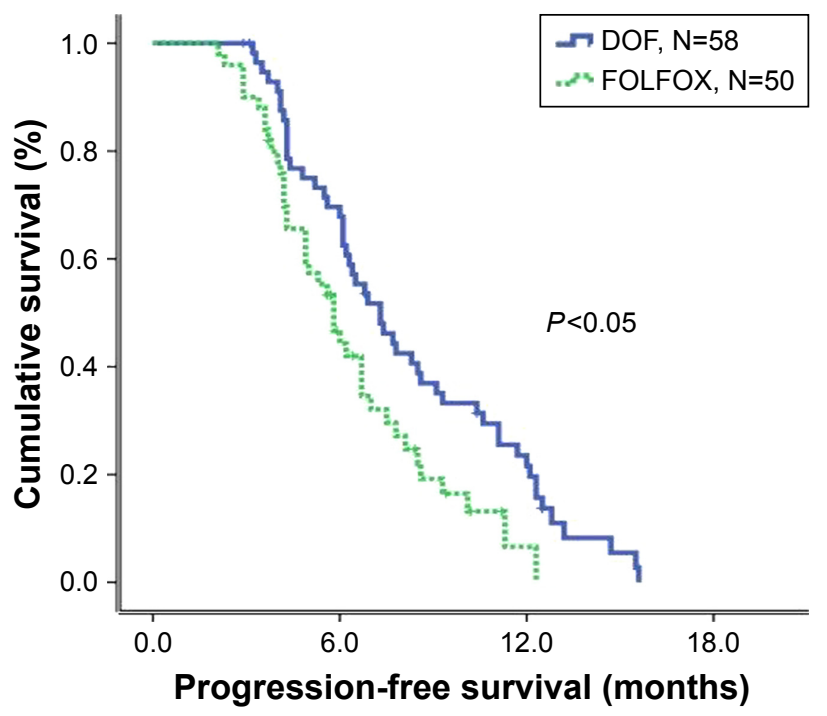

Figure I The Kaplan-Meier curve for progression-free survival.

Notes: Kaplan-Meier analysis showed that advanced gastric cancer patients on DOF regimen had longer progression-free survival than patients with FOLFOX regimen ( 8.2 versus 6.4 months; $P=0.01 \mathrm{I}$ ).

Abbreviations: DOF, docetaxel, oxaliplatin, and fluorouracil; FOLFOX, oxaliplatin, fluorouracil, and leucovorin.

tumor stage, and metastatic sites were also independent prognostic factors (Table 3). The Cox-regression analysis for PFS showed that response, metastatic sites, and age were independent prognostic factors for PFS (Table 4).

\section{Toxicity}

The toxicity analysis is shown in Table 5. The toxicities of the two regimens were all manageable, and total toxicity $(P=0.071)$ and grade $3-4$ toxicity $(P=0.247)$ had no differences. Grade 3-4 adverse events included

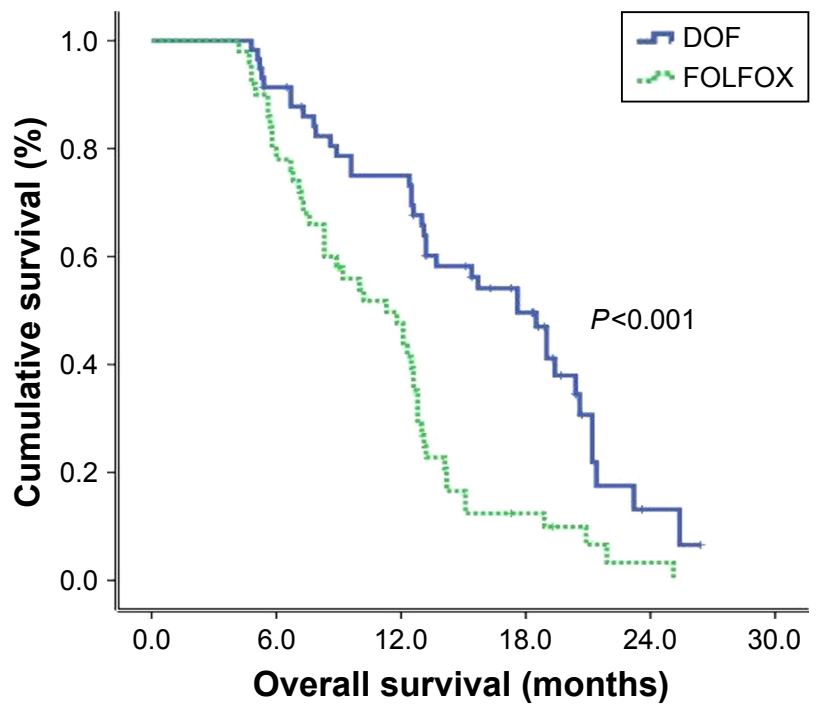

Figure 2 The Kaplan-Meier curve for overall survival.

Notes: Kaplan-Meier analysis showed that advanced gastric cancer patients on DOF regimen had longer overall survival than patients with FOLFOX regimen ( 16.3 versus II.2 months; $P<0.00$ I).

Abbreviations: DOF, docetaxel, oxaliplatin, and fluorouracil; FOLFOX, oxaliplatin, fluorouracil, and leucovorin.
Table 3 Multivariate analysis of overall survival

\begin{tabular}{llllll}
\hline & Wald $\chi^{2}$ & OR & \multicolumn{2}{c}{$\mathbf{9 5 \%}$ Cl for OR } & P-value \\
\cline { 4 - 5 } & & & Lower & Upper & \\
\hline Regimen & 11.899 & 2.456 & 1.474 & 4.092 & 0.001 \\
Age & 2.761 & 0.653 & 0.395 & 1.080 & 0.097 \\
Sex & 0.530 & 0.786 & $0.41 \mathrm{I}$ & 1.502 & 0.467 \\
ECOG & 2.531 & 0.733 & 0.501 & 1.075 & 0.112 \\
Clinical stage & 5.478 & 0.385 & 0.173 & 0.856 & 0.019 \\
Tumor location & 3.146 & 0.834 & 0.683 & 1.019 & 0.076 \\
Metastasis & 5.557 & 1.364 & 1.054 & 1.765 & 0.018 \\
Ascites & 0.096 & 1.102 & 0.598 & 2.031 & 0.756 \\
Response & 11.925 & 1.737 & 1.270 & 2.377 & 0.001
\end{tabular}

Abbreviations: ECOG, Eastern Cooperative Oncology Group performance status; OR, odds ratio.

thrombocytopenia, anemia, febrile neutropenia, neutropenia, stomatitis, diarrhea, nausea/vomiting, and peripheral neuropathy. In the DOF group, the incidence of grade 3-4 peripheral neuropathy was significantly higher than that in the FOLFOX group $(10.3 \%$ versus $2 \% ; P<0.05)$. The rates of grade $3-4$ neutropenia and thrombocytopenia were both relatively high in the two groups (19\% versus $12 \% ; P>0.05,10.3 \%$ versus $6.0 \% ; P>0.05)$. However, the FOLFOX regimen induced less toxicity than the DOF regimen in patients older than 65 years; the DOF regimen was associated with more neutropenia ( $67 \%$ versus $30 \% ; P<0.05$ ), thrombocytopenia (61\% versus $52 \% ; P<0.05)$, and peripheral neuropathy ( $49 \%$ versus $22 \% ; P<0.05)$.

Dose reduction, dose delays, and treatment-related mortality were similar between the two arms. There was no treatment-related mortality in the DOF and FOLFOX groups (data not shown).

\section{Discussion}

Gastric cancer is one of the most common malignancies in China; despite the fact that incidence and mortality rates have declined over the last two decades, over $40 \%$ of gastric cancer patients were at an advanced stage at the time of diagnosis. ${ }^{2}$

Table 4 Multivariate analysis of progression-free survival

\begin{tabular}{llllll}
\hline & Wald $\chi^{2}$ & OR & \multicolumn{2}{c}{$\mathbf{9 5 \%} \mathbf{C l}$ for OR } & P-value \\
\cline { 4 - 5 } & & & Lower & Upper & \\
\hline Regimen & 1.319 & 1.339 & 0.814 & 2.204 & 0.251 \\
Age & 6.038 & 0.550 & 0.341 & 0.886 & 0.014 \\
Sex & 0.987 & 0.753 & 0.429 & 1.319 & 0.320 \\
ECOG & 0.539 & 1.145 & 0.797 & 1.645 & 0.463 \\
Clinical stage & 0.429 & 0.761 & 0.336 & 1.723 & 0.513 \\
Tumor location & 1.190 & 0.902 & 0.748 & 1.086 & 0.275 \\
Metastasis & 5.355 & 1.319 & 1.043 & 1.668 & 0.021 \\
Ascites & 3.158 & 0.599 & 0.340 & 1.054 & 0.076 \\
Response & 14.924 & 1.800 & 1.336 & 2.425 & 0.000 \\
\hline
\end{tabular}

Abbreviations: ECOG, Eastern Cooperative Oncology Group performance status; OR, odds ratio. 
Table 5 Toxic effects according to National Cancer Institute common toxicity criteria

\begin{tabular}{|c|c|c|c|c|}
\hline \multirow[t]{3}{*}{ Adverse events } & \multicolumn{4}{|c|}{ Chemotherapeutic regimen } \\
\hline & \multicolumn{2}{|c|}{ DOF, N=58 n (\%) } & \multicolumn{2}{|c|}{ FOLFOX, N=50 n (\%) } \\
\hline & All grades & Grade 3-4 & All grades & Grade 3-4 \\
\hline \multicolumn{5}{|l|}{ Nonhematological } \\
\hline Nausea or vomiting & $45(77.6)$ & $5(8.6)$ & $36(72.0)$ & $6(12.0)$ \\
\hline Diarrhea & $15(25.9)$ & $3(5.2)$ & $26(52.0)$ & $2(4.0)$ \\
\hline Stomatitis & $18(31.0)$ & $2(3.4)$ & $6(12.0)$ & I $(2.0)$ \\
\hline Peripheral neuropathy & II (I9.0) & $6(10.3)$ & $4(8.0)$ & I (2.0) \\
\hline \multicolumn{5}{|l|}{ Hematological } \\
\hline Neutropenia & $26(44.8)$ & I (I9.0) & $25(50.0)$ & $6(12.0)$ \\
\hline Febrile neutropenia & $6(10.3)$ & I (I.7) & $7(14.0)$ & $0(0.0)$ \\
\hline Anemia & $29(50.0)$ & $0(0.0)$ & $31(62.0)$ & $2(4.0)$ \\
\hline Thrombocytopenia & $21(36.2)$ & $6(10.3)$ & $15(30.0)$ & $3(6.0)$ \\
\hline
\end{tabular}

Abbreviations: DOF, docetaxel, oxaliplatin, and fluorouracil; FOLFOX, oxaliplatin, fluorouracil, and leucovorin.

The prognosis for AGC remains unsatisfactory with a 5-year survival rate $<10 \%$ and median OS $<1$ year. ${ }^{26}$ For patients with AGC who cannot receive surgery or develop postoperative recurrent metastasis, chemotherapy has become an indispensable treatment. The current research has been focusing on finding a better combination to improve the outcomes while minimizing the toxicity.

In China, the FOLFOX regimen has been used widely for AGC. In several studies conducted over the last decade, different FOLFOX regimens have exhibited satisfactory clinical activity and acceptable toxicity. The FOLFOX-6 (oxaliplatin $100 \mathrm{mg} / \mathrm{m}^{2}$ and leucovorin $400 \mathrm{mg} / \mathrm{m}^{2}$ [2 hour intravenous infusion] followed by a $5-\mathrm{FU}$ bolus of $400 \mathrm{mg} / \mathrm{m}^{2}$ [10 min infusion] and then $5-\mathrm{FU} 2,600-3,000 \mathrm{mg} / \mathrm{m}^{2}$ [46 hour continuous infusion]. Repeated every 14 days) regimen was reported with an ORR of $40.2 \%-48 \%$, a TTP of 5.4-6.2 months, and an OS of 8.6-13 months. ${ }^{27,28}$ The mFOLFOX-6 (modified FOLFOX-6; $85 \mathrm{mg} / \mathrm{m}^{2}$ oxaliplatin and $400 \mathrm{mg} / \mathrm{m}^{2}$ leucovorin as a 2 hour infusion, followed by a 5 -FU bolus of $400 \mathrm{mg} / \mathrm{m}^{2}$ and $2,400 \mathrm{mg} / \mathrm{m}^{2} 5$-FU as a 46 hour continuous infusion. Repeated every 14 days) regimen exhibited good efficacy, with an ORR of $37.0 \%$, a median TTP of 6.5 months, and a median OS of 11.4 months. ${ }^{29}$ Our present study was basically consistent with these results investigating the FOLFOX regimen.

In addition, docetaxel is an effective agent for AGC, and the TAX325 trial established docetaxel as a new option for the treatment of metastatic gastric cancer. ${ }^{30,31}$ The results of the V325 trial demonstrated that adding docetaxel to the $\mathrm{CF}$ (cisplatin and 5-FU) regimen not only resulted in a higher response rate, but also a prolonged TTP and OS, and the quality of life was improved as well. ${ }^{11}$ Furthermore, Al-Batran et al reported an improved median PFS of 9 months and a median OS of 17.3 months with FLOT (5-FU, leucovorin, oxaliplatin and docetaxel). ${ }^{32}$ The results of a Phase II study by Van Cutsem showed that in AGC patients treated with TEF (docetaxel plus oxaliplatin and 5-FU) regimen, the median OS of $>14$ months was substantially better than the 8-9 months reported in previous international multicenter studies. ${ }^{33}$

To our knowledge, although there had been some research on the DOF regimen before our study, those studies were performed in the Western population. It is unknown whether the DOF regimen is appropriate for East Asian patients. Therefore, we performed the present study to investigate the safety and efficacy of the DOF regimen compared to that of the FOLFOX regimen for treating Chinese patients with locally AGC.

In this study, we investigated the efficacy and safety of FOLFOX and DOF as first-line regimens in AGC. In terms of results, the PFS and OS were better in the DOF group than the FOLFOX group, and the differences were significant statistically ( 8.2 versus 6.4 months; $P<0.05,16.3$ versus 11.2 months; $P<0.001)$. The multivariate Cox-regression analysis showed that regimen, response, tumor stage, and metastatic sites were independent prognostic factors for OS. And response, metastatic sites, and age were independent prognostic factors for PFS.

With regard to toxicity, the incidences of toxicity of the two regimens were manageable, and total toxicity $(P=0.071)$ and grade $3-4$ toxicity $(P=0.247)$ had no differences. In the DOF group, the incidence of grade 3-4 peripheral neuropathy was significantly higher than that in the FOLFOX group $(10.3 \%$ versus $2 \%, P<0.05)$. However, the rate of neuropathy was lower in our study than that reported in the literature for both the groups. This may be explained by the use of G-CSF in primary or secondary prophylaxis during chemotherapy.

In the subgroup analysis of the present study, the DOF regimen resulted in a better PFS (8.2 versus 6.4 months; 
$P=0.038)$ and $\mathrm{OS}(15.3$ versus 9.8 months; $P=0.004)$ than FOLFOX, in patients older than 65 years. However, the FOLFOX regimen had reduced toxicity compared with DOF, and the DOF regimen was associated with more neutropenia (67\% versus $30 \% ; P<0.05)$, thrombocytopenia (61\% versus $52 \% ; P<0.05$ ), and peripheral neuropathy (49\% versus $22 \%$; $P<0.05)$. The results of a recent study in elderly patients ( $\geq 65$ years) with gastroesophageal carcinoma suggested that docetaxel-containing triplet regimens were feasible in elderly patients with gastroesophageal cancer. However, toxicity was significantly increased and quality of life deteriorated in patients aged 70 years or older. ${ }^{32}$ Therefore, this regimen should be recommended only in selected fit patients, if they are elderly. In this regard, a suitable age may be relatively difficult to ascertain when choosing a FOLFOX regimen for older patients, because the overall situation, such as performance status and sensitivity to chemotherapy, should be taken into consideration.

In conclusion, the present study demonstrated that the DOF regimen was more effective than the FOLFOX regimen for patients with locally $\mathrm{AGC}$, both in younger and elderly patients ( $\geq 65$ years). The adverse effects of the two regimens were tolerated by administering supportive treatment. In elderly patients, the toxicity analysis of the DOF regimen was associated with worse nonhematological toxicities; therefore, considering that the goals of palliative chemotherapy are to prolong and preserve quality of life, the FOLFOX regimen may be a more suitable option for elderly patients with AGC.

Our study had several limitations because it was a retrospective and nonrandomized study. First, the proportion of patients with an ECOG PS of 2 was significantly higher in the FOLFOX group, although PS is not an accurate criterion for evaluating the general status of cancer patients. Second, there was heterogeneity in the DOF arm of the study in terms of primary prophylaxis at the physician's discretion. Besides, the sample size was small and we collected data only from a single institution. Therefore, a larger prospective randomized Phase III trial in several institutions is expected to be conducted to further verify our results in the future.

\section{Acknowledgments}

We thank all the people who participated in this study.

\section{Disclosure}

The authors report no conflicts of interest in this work.

\section{References}

1. Jemal A, Bray F, Center MM, Ferlay J, Ward E, Forman D. Global cancer statistics. CA Cancer J Clin. 2011;61(2):69-90.

2. Siegel R, Ma J, Zou Z, Jemal A. Cancer statistics, 2014. CA Cancer J Clin. 2014;64(1):9-29.

3. Glimelius B, Ekstrom K, Hoffman K, et al. Randomized comparison between chemotherapy plus best supportive care with best supportive care in advanced gastric cancer. Ann Oncol. 1997;8(2):163-168.

4. Wagner AD, Grothe W, Haerting J, Kleber G, Grothey A, Fleig WE. Chemotherapy in advanced gastric cancer: a systematic review and meta-analysis based on aggregate data. J Clin Oncol. 2006;24(18):2903-2909.

5. Casaretto L, Sousa PL, Mari JJ. Chemotherapy versus support cancer treatment in advanced gastric cancer: a meta-analysis. Braz J Med Biol Res. 2006;39(4):431-440.

6. Wagner $\mathrm{AD}$, Unverzagt $\mathrm{S}$, Grothe $\mathrm{W}$, et al. Chemotherapy for advanced gastric cancer. Cochrane Database Syst Rev. 2010;3:CD004064.

7. Iwase H, Shimada M, Tsuzuki T, et al. A phase II multi-center study of triple therapy with paclitaxel, $\mathrm{S}-1$ and cisplatin in patients with advanced gastric cancer. Oncology. 2011;80(1-2):76-83.

8. Koizumi W, Nakayama N, Tanabe S, et al. A multicenter phase II study of combined chemotherapy with docetaxel, cisplatin, and S-1 in patients with unresectable or recurrent gastric cancer (KDOG 0601). Cancer Chemother Pharmacol. 2012;69(2):407-413.

9. Kim JY, Do YR, Park KU, et al. Multicenter phase II trial of S-1, paclitaxel and cisplatin triplet combination chemotherapy in patients with advanced gastric cancer. Cancer Chemother Pharmacol. 2011;67(3): $527-532$.

10. Koizumi W, Narahara H, Hara T, et al. S-1 plus cisplatin versus S-1 alone for first-line treatment of advanced gastric cancer (SPIRITS trial): a phase III trial. Lancet Oncol. 2008;9(3):215-221.

11. Van Cutsem E, Moiseyenko VM, Tjulandin S, et al; V325 Study Group. Phase III study of docetaxel and cisplatin plus fluorouracil compared with cisplatin and fluorouracil as first-line therapy for advanced gastric cancer: a report of the V325 Study Group. J Clin Oncol. 2006;24(31):4991-4997.

12. Ajani JA, Moiseyenko VM, Tjulandin S, et al; V-325 Study Group. Clinical benefit with docetaxel plus fluorouracil and cisplatin compared with cisplatin and fluorouracil in a phase III trial of advanced gastric or gastroesophageal cancer adenocarcinoma: the V-325 Study Group. J Clin Oncol. 2007;25(22):3205-3209.

13. Ajani JA, Moiseyenko VM, Tjulandin S, et al; V-325 Study Group. Quality of life with docetaxel plus cisplatin and fluorouracil compared with cisplatin and fluorouracil from a phase III trial for advanced gastric or gastroesophageal adenocarcinoma: the V-325 Study Group. J Clin Oncol. 2007;25(22):3210-3216.

14. Hwang IG, Ji JH, Kang JH, et al. A multi-center, open-label, randomized phase III trial of first-line chemotherapy with capecitabine monotherapy versus capecitabine plus oxaliplatin in elderly patients with advanced gastric cancer. J Geriatr Oncol. 2017;8(3):170-175.

15. Al-Batran SE, Hartmann JT, Probst S, et al; Arbeitsgemeinschaft Internistische Onkologie. Phase III trial in metastatic gastroesophageal adenocarcinoma with fluorouracil, leucovorin plus either oxaliplatin or cisplatin: a study of the Arbeitsgemeinschaft Internistische Onkologie. J Clin Oncol. 2008;26(9):1435-1442.

16. Cunningham D, Starling N, Rao S, et al; Upper Gastrointestinal Clinical Studies Group of the National Cancer Research Institute of the United Kingdom. Capecitabine and oxaliplatin for advanced esophagastric cancer. N Engl J Med. 2008;358(1):36-46.

17. Al-Batran SE, Hartmann JT, Hofheinz R, et al. Biweekly fluorouracil, leucovorin, oxaliplatin, and docetaxel (FLOT) for patients with metastatic adenocarcinoma of the stomach or resophagogastric junction: a phase II trial of the Arbeitsgemeinschaft Internistische Onkologie. Ann Oncol. 2008;19(11):1882-1887.

18. Louvet C, Andre T, Tigaud JM, et al. Phase II study of oxaliplatin, fluorouracil, and folinic acid in locally advanced or metastatic gastric cancer patients. J Clin Oncol. 2002;20(23):4543-4548. 
19. Al-Batran SE, Atmaca A, Hegewisch-Becker S, et al. Phase II trial of biweekly infusional fluorouracil, folinic acid, and oxaliplatin in patients with advanced gastric cancer. J Clin Oncol. 2004;22(4):658-663.

20. Zhang J, Chen RX, Zhang J, et al. Efficacy and safety of neoadjuvant chemotherapy with modified FOLFOX7 regimen on the treatment of advanced gastric cancer. Chin Med J (Engl). 2012;125(12): 2144-2150.

21. Yeh YS, Tsai HL, Ma CJ, et al. A retrospective study of the safety and efficacy of a first-line treatment with modified FOLFOX-4 in unresectable advanced or recurrent gastric cancer patients. Chemotherapy. 2012;58(5):411-418.

22. Catalano V, Bisonni R, Graziano F, et al. A phase II study of modified FOLFOX as first-line chemotherapy for metastatic gastric cancer in elderly patients with associated diseases. Gastric Cancer. 2013; 16(3):411-419.

23. Chen SS, Yang XC, Chi F, et al. A phase II study of preoperative chemotherapy with modified FOLFOX6 followed by surgery and postoperative chemoradiation in patients with localized gastric adenocarcinoma. Oncol Res. 2013;20(7):327-332.

24. Eisenhauer EA, Therasse P, Bogaerts J, et al. New response evaluation criteria in solid tumours: revised RECIST guideline (version 1.1). Eur J Cancer. 2009;45(2):228-247.

25. Cirillo M, Venturini M, Ciccarelli L, Coati F, Bortolami O, Verlato G. Clinician versus nurse symptom reporting using the National Cancer Institute-Common Terminology Criteria for Adverse Events during chemotherapy: results of a comparison based on patient's self-reported questionnaire. Ann Oncol. 2009;20(12):1929-1935.
26. Power DG, Kelsen DP, Shah MA. Advanced gastric cancer slow but steady progress. Cancer Treat Rev. 2010;36(5):384-392.

27. Luo HY, Xu RH, Zhang L, et al. A pilot study of oxaliplatin, fluorouracil and folinic acid (FOLFOX-6) as first-line chemotherapy in advanced or recurrent gastric cancer. Chemotherapy. 2008;54(3):228-235.

28. Lee HH, Hur H, Kim SH, Park AR, Kim W, Jeon HM. Outcomes of modified FOLFOX-6 as first line treatment in patients with advanced gastric cancer in a single institution; retrospective analysis. Cancer Res Treat. 2010;42(1):18-23.

29. Hacibekiroglu I, Kodaz H, Erdogan B, et al. Comparative analysis of the efficacy and safety of modified FOLFOX-6 and DCF regimens as first-line treatment in advanced gastric cancer. Mol Clin Oncol. 2015; 3(5):1160-1164.

30. Lordick F, Lorenzen S, Yamada Y, Ilson D. Optimal chemotherapy for advanced gastric cancer: is there a global consensus? Gastric Cancer. 2014;17(2):213-225.

31. Nishiyama M, Wada S. Docetaxel: its role in current and future treatments for advanced gastric cancer. Gastric Cancer. 2009;12(3):132-141.

32. Al-Batran SE, Pauligk C, Homann N, et al. The feasibility of triple-drug chemotherapy combination in older adult patients with oesophagogastric cancer: a randomised trial of the Arbeitsgemeinschaft Internistische Onkologie (FLOT65+). Eur J Cancer. 2013;49(4):835-842.

33. Van Cutsem E, Boni C, Tabernero J, et al. Docetaxel plus oxaliplatin with or without fluorouracil or capecitabine in metastatic or locally recurrent gastric cancer: a randomized phase II study. Ann Oncol. 2015;26(1):149-156.
OncoTargets and Therapy

\section{Publish your work in this journal}

OncoTargets and Therapy is an international, peer-reviewed, open access journal focusing on the pathological basis of all cancers, potential targets for therapy and treatment protocols employed to improve the management of cancer patients. The journal also focuses on the impact of management programs and new therapeutic agents and protocols on

\section{Dovepress}

patient perspectives such as quality of life, adherence and satisfaction. The manuscript management system is completely online and includes a very quick and fair peer-review system, which is all easy to use. Visit http://www.dovepress.com/testimonials.php to read real quotes from published authors. 